\title{
Perfil funcional respiratório de crianças com COVID-19 durante a fase de
}

\section{hospitalização}

\author{
Respiratory functional profile of children with COVID-19 during the hospitalization phase \\ Perfil funcional respiratorio de niños con COVID-19 durante la fase de hospitalización
}

Recebido: 01/08/2021 | Revisado: 06/08/2021 | Aceito: 17/08/2021 | Publicado: 19/08/2021

\author{
Davi Santana Sousa \\ ORCID: https://orcid.org/0000-0001-7103-4441 \\ Universidade Tiradentes, Brasil \\ Universidad Popular Autónoma del Estado de Puebla, México \\ E-mail: davi.santana.sousa@hotmail.com \\ Jordana Borges Brota \\ ORCID: https://orcid.org/0000-0002-0760-4064 \\ Universidade Tiradentes, Brasil \\ E-mail: brotajordana@gmail.com \\ Rebeca Maria Santos Araujo \\ ORCID: https://orcid.org/0000-0002-4997-8240 \\ Universidade Tiradentes, Brasil \\ E-mail: rebecaaraujo_20@hotmail.com \\ Aida Carla Santana de Melo Costa \\ ORCID: https://orcid.org/0000-0002-4192-7887 \\ Universidade Tiradentes, Brasil \\ E-mail: aida-fisio@hotmail.com
}

\begin{abstract}
Resumo
A COVID-19 é uma doença derivada do novo coronavírus SARS-CoV-2, que afeta todas as faixas etárias de forma leve, moderada ou grave e que pode requerer cuidados hospitalares. Os principais sinais e sintomas assemelham-se a uma gripe ou resfriado. Nas crianças, é comum a presença de tosse, febre, desconforto respiratório, náuseas, vômitos e ausculta pulmonar com creptos e roncos. Em todo o mundo é encontrada uma menor incidência dos casos em crianças, sendo bastante discutida a justificativa para tal condição. Este trabalho justifica-se pelo período pandêmico atual, o qual suscitou o interesse em investigar o perfil funcional respiratório na população pediátrica do Hospital de Urgência de Sergipe (HUSE), a fim de promover descobertas sobre as manifestações clínicas da COVID-19 em crianças, bem como suas complicações. O objetivo geral desta pesquisa foi estabelecer o perfil funcional respiratório das crianças com COVID-19, admitidas no serviço de pediatria do Hospital de Urgência de Sergipe. Trata-se de um estudo observacional, do tipo analítico, transversal e de campo, com abordagem quantitativa, realizado no período de julho a setembro de 2020. Observou-se que a maioria dos pacientes necessitou de suporte ventilatório, apresentava secreções brônquicas e evoluiu com pneumonia. Não foram encontradas discrepâncias nos achados em relação aos sinais e sintomas apresentados pelos adultos, porém evidenciou-se que a doença acontece em menor incidência e de forma mais branda.
\end{abstract}

Palavras-chave: Criança; Hospitalização; Perfil de Impacto da doença.

\begin{abstract}
COVID-19 is a disease derived from the new coronavirus SARS-CoV-2, which affects all age groups in a mild, moderate or severe manner and which may require hospital care. The main signs and symptoms are similar to flu or cold. In children, cough, fever, respiratory distress, nausea, vomiting and changes in pulmonary auscultation are common, such as crackle and snoring. A lower incidence of cases in children is find over the world and the justification for this is widely discussed. This work is justified by the current pandemic period, which aroused the interest in investigating the respiratory functional profile in the pediatric population at Sergipe Emergency Hospital, in order to promote discoveries about the clinical manifestations of COVID-19 in children, as well as their complications. The general objective of this research was to establish the functional respiratory profile of children with COVID-19, admitted to pediatric service at Sergipe Emergency Hospital. This is an observational, analytical, cross-sectional and field study, with a quantitative approach, accomplished from July to September 2020. It was observe that the most patients needed oxygen support, they were secretive and evolved with pneumonia. There were not discrepancies in the findings in relation to signs and symptoms presented by adults, however it was show that the disease occurs in a lower incidence and in a milder way.
\end{abstract}

Keywords: Child; Hospitalization; Disease impact profile. 


\begin{abstract}
Resumen
COVID-19 es una enfermedad derivada del nuevo coronavirus SARS-CoV-2, que afecta a todos los grupos de edad de manera leve, moderada o grave y puede requerir atención hospitalaria. Los principales signos y síntomas se asemejan a los de una gripe o un resfriado. En los niños es común tos, fiebre, molestias respiratorias, náuseas, vómitos y auscultación pulmonar con crepitantes y ronquidos. Se encuentra una menor incidencia de casos en niños en todo el mundo, y la justificación de esta condición se discute ampliamente. Este trabajo se justifica por el actual período pandémico, que despertó el interés por investigar el perfil funcional respiratorio en la población pediátrica del Hospital de Urgência de Sergipe (HUSE), con el fin de promover descubrimientos sobre las manifestaciones clínicas del COVID-19 en niños. , así como sus complicaciones. El objetivo general de esta investigación fue establecer el perfil funcional respiratorio de los niños con COVID-19 ingresados en el servicio de pediatría del Hospital de Urgência de Sergipe. Se trata de un estudio observacional, analítico, transversal y de campo, con abordaje cuantitativo, realizado de julio a septiembre de 2020. Se observó que la mayoría de los pacientes necesitaban soporte ventilatorio, presentaban secreciones bronquiales y evolucionaban con neumonía. No hubo discrepancias en los hallazgos en relación a los signos y síntomas que presentan los adultos, pero se demostró que la enfermedad se presenta con menor incidencia y de forma más leve.
\end{abstract}

Palabras clave: Niño; Hospitalización; Perfil de impacto de la enfermedad.

\title{
1. Introdução
}

Coronavírus constituem uma grande família de vírus comuns que afetam diferentes espécies de animais, como camelos, gado, gatos e morcegos. Raramente esses vírus que infectam animais podem infectar pessoas, como, por exemplo, MERS-CoV e SARS-CoV. Recentemente, em dezembro de 2019, houve a transmissão de um novo coronavírus (SARS-CoV2), o qual foi identificado primeiramente em Wuhan na China e causou a COVID-19, a qual foi disseminada e transmitida de pessoa a pessoa em todo o mundo. Tal doença é causada pelo coronavírus, denominado SARS-CoV-2, que apresenta um espectro clínico variando de infecções assintomáticas a quadros graves (Guedes et al., 2020; Ministério da Saúde, 2020).

Segundo a Organização Mundial de Saúde (OMS), cerca de 80\% dos pacientes infectados pela COVID-19 podem ser assintomáticos ou oligossintomáticos (manifestação de poucos sintomas) e, aproximadamente, $20 \%$ dos casos detectados necessitam de atendimento hospitalar por apresentarem dificuldade respiratória. Dentre eles, quase 5\% podem necessitar de suporte ventilatório (Pereira et al., 2020; Ministério da Saúde, 2020).

O SARS-CoV-2 apresenta sintomas que são semelhantes a um resfriado ou a uma gripe. Os sintomas mais frequentes em crianças são desconforto respiratório, febre, tosse, vômitos e náuseas. Na ausculta pulmonar, observa-se redução do murmúrio vesicular e presença de crepitações. Crianças e jovens representam apenas 1-2\% dos casos de COVID-19 em todo o mundo. No Estado de Sergipe, a incidência é de 0,1\% das crianças menores de 1 ano; 1,2\% entre 1 e 4 anos; $2,8 \%$ de 5 a 14 anos e 3,6\% de 15 a 19 anos (Oliveira et al., 2020; Governo do Estado de Sergipe, 2020).

A hipótese mais frequente e mais interessante para a menor gravidade da COVID-19 em crianças pode estar relacionada à expressão da enzima conversora de angiotensina 2 (ACE2) nas células epiteliais alveolares do tipo I e II. Verificou-se que a ACE2 é o receptor do SARS-CoV-2, e é necessária para a entrada na célula hospedeira e subsequente replicação do vírus. Por essa razão, uma quantidade limitada da ACE2 na infância, em um período em que os pulmões ainda estão em desenvolvimento, poderia proteger as crianças de formas graves da doença (Safadi, 2020).

Outra hipótese é que crianças menores de 2 anos de idade têm cinco ou mais infecções respiratórias por ano e permanecem uma média de 44 dias com doenças respiratórias leves. A ativação da imunidade adaptativa para coronavírus comuns, assim como a ativação do sistema imunológico inato no trato respiratório, podem realmente fornecer alguma proteção contra a infecção microbiana, incluindo SARS-CoV-2. As infecções respiratórias frequentes nas crianças podem fornecer mais pistas sobre por que são resistentes. As crianças, muitas vezes, são infectadas por mais de um agente viral. A interferência viral é um fenômeno bem conhecido em que um vírus interfere na replicação de um segundo vírus. Existem algumas evidências de coinfecções em pacientes com COVID-19, incluindo coinfecção com outros coronavírus (Steinman et al., 2020).

O SARS-CoV-2, associado a resfriados comuns e gripe, induz a uma regulação negativa de ACE2. Ou seja, uma 
redução no receptor viral para SARS-CoV-2, o que pode ajudar a explicar por que as crianças que carregam esses vírus em seu nariz e porções de suas vias respiratórias superiores são hospitalizadas com menos frequência do que os adultos (Steinman et al., 2020; Smile, 2020).

A terceira hipótese mais discutida é que a resposta da célula $\mathrm{T}$ desempenha um papel protetor importante contra vírus respiratórios. A imunidade mediada por células T é crucial para controlar a replicação do vírus e a gravidade da COVID-19. A profunda linfocitopenia observada em adultos, agravada pelos efeitos do envelhecimento na adaptação à resposta imune, pode desempenhar um papel no aumento da carga viral de SARS-CoV-2 em adultos, quando comparados a crianças (Lingappan et al., 2020).

Este trabalho justifica-se pelo período pandêmico atual, o qual suscitou o interesse em investigar o perfil funcional respiratório na população pediátrica do Hospital de Urgência de Sergipe, a fim de promover descobertas sobre as manifestações clínicas da COVID-19 em crianças, bem como suas complicações.

Neste sentido, o objetivo geral desta pesquisa foi estabelecer o perfil funcional respiratório das crianças com COVID19, admitidas no serviço de pediatria do Hospital de Urgência de Sergipe.

\section{Metodologia}

\subsection{Delineamento da Pesquisa}

Trata-se de um estudo observacional, do tipo analítico, transversal e de campo, com abordagem quantitativa.

\subsection{Local da Pesquisa e Caracterização}

A pesquisa foi realizada no internamento pediátrico do Hospital de Urgência de Sergipe (HUSE). Este hospital constitui a maior unidade hospitalar pública do Estado, a qual oferece diversos serviços prestados à população sergipana e aos usuários do Sistema Único de Saúde (SUS) de estados circunvizinhos, como Bahia e Alagoas, que são encaminhados para essa unidade por conter um serviço de alta complexidade.

\subsection{Casuística}

A amostra foi selecionada por conveniência, obtida por meio da coleta de dados realizada durante o período de três meses, de julho a setembro de 2020, perfazendo um total de dezesseis participantes, sendo incluídas as crianças acometidas pela COVID-19.

\subsection{Aspectos Éticos}

O estudo cumpriu os termos da Resolução 466/12, de 12 de dezembro de 2012, do Conselho Nacional de Saúde do Ministério de Saúde foram respeitados. O Termo de Consentimento Livre e Esclarecido (TCLE) foi elaborado pelos pesquisadores e entregue aos participantes da pesquisa, via online, para que os mesmos pudessem ler e assinar consentindo a sua participação.

\subsection{Instrumentos E Procedimentos Para Coleta De Dados}

A coleta de dados foi feita mediante análise dos prontuários dos pacientes internados na enfermaria pediátrica, admitidos no período de julho a setembro de 2020 no Hospital de Urgência de Sergipe. Foram registradas as informações contidas nos prontuários quanto à idade, sexo, ausculta pulmonar, saturação parcial de oxigênio, suporte ventilatório, tipo de suporte, presença de secreção em vias aéreas, instalação de quadro de pneumonia e desfecho clínico. 


\subsection{Análise Estatística}

Os dados deste estudo foram analisados de forma descritiva e analítica. As variáveis numéricas foram observadas quanto à distribuição de normalidade por meio do teste de Shapiro-Wilk. Nos casos em que os pressupostos foram atendidos, as mesmas foram apresentadas por meio de média (x) e desvio padrão (DP). Caso contrário, por meio de mediana (Md) e seus quartis $\left(1^{\circ}-3^{\circ}\right)$. As variáveis categóricas foram apresentadas por meio de frequência absoluta e relativa. A significância estatística foi adotada em $5 \%(\mathrm{p} \leq 0,05)$.

\section{Resultados}

Fizeram parte do estudo 16 crianças, $10(62,5 \%)$ do sexo masculino e $6(37,5 \%)$ do sexo feminino. A idade foi apresentada por mediana, sendo de $4(0,1$ - 9) meses. Considerando a variável Suporte de Oxigênio, observou-se que $75 \%$ dos pacientes fizeram uso de algum tipo de suporte ventilatório, enquanto apenas $25 \%$ mantiveram-se em ar ambiente. Referente à presença de secreções, $62,5 \%$ dos pacientes encontravam-se com secreções brônquicas e 37,5\% não apresentavam secreções em vias aéreas. Das crianças avaliadas, 62,5\% evoluíram com Pneumonia. Do total de pacientes do estudo, 87,5\% receberam alta, enquanto $12,5 \%$ foram a óbito (Tabela 1).

Tabela 1. Caracterização da Amostra dos pacientes pediátricos internados por COVID-19.

\begin{tabular}{ll}
\hline Sexo & Total de Pacientes (\%) \\
\hline Feminino & $37,5 \%$ \\
Masculino & $62,5 \%$ \\
Suporte de O2 & Total de Pacientes (\%) \\
Sim & $75 \%$ \\
Não & $25 \%$ \\
Presença de Secreção & Total de Pacientes (\%) \\
Sim & $37,5 \%$ \\
Não & $62,5 \%$ \\
Pneumonia & Total de Pacientes (\%) \\
Presente & $62,5 \%$ \\
Ausente & $37,5 \%$ \\
Desfecho do Paciente & Total de Pacientes (\%) \\
Alta & $87,5 \%$ \\
Óbito & $12,5 \%$ \\
\hline
\end{tabular}

Fonte: Autores.

Quanto à ausculta pulmonar, 31,25\% da amostra não apresentaram ruídos adventícios, enquanto 31,25\% apresentaram estertores crepitantes, $25 \%$ manifestaram roncos e 12,5\% possuíam associação de roncos e estertores crepitantes (Figura 1). 
Figura 1. Caracterização da Ausculta Pulmonar apresentada pelos pacientes pediátricos internados por COVID-19.

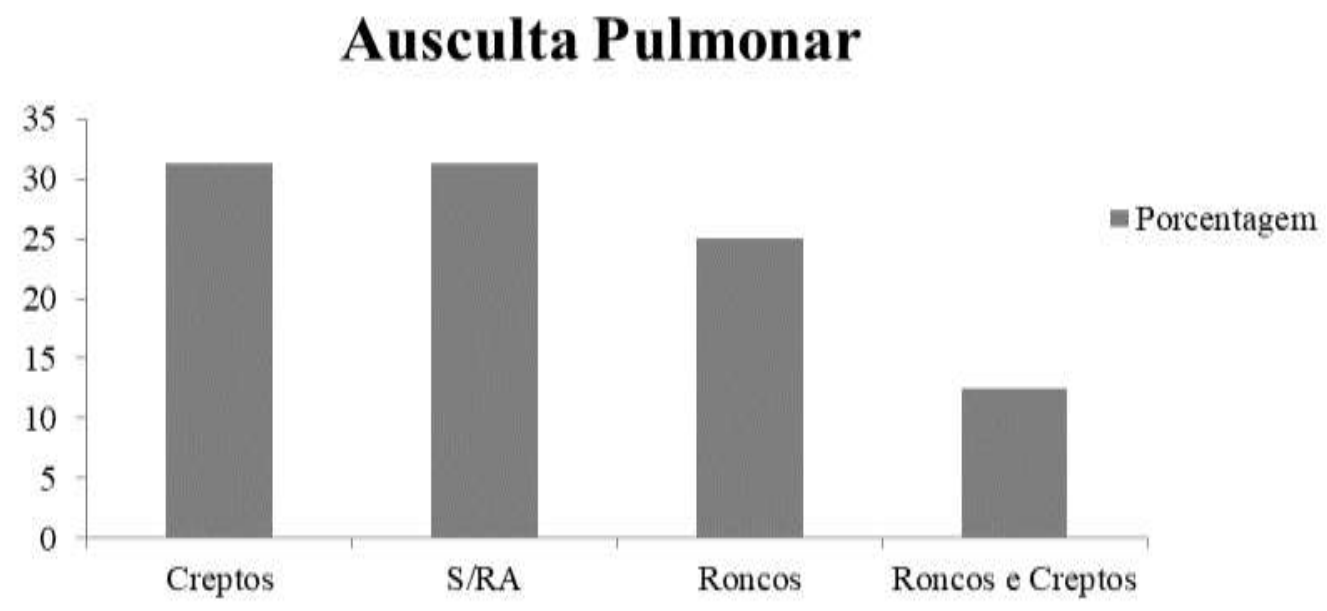

Fonte: Autores.

Em relação ao tipo de suporte ventilatório utilizado, 33,3\% utilizaram Halo de Oxigênio, 33,33\% fizeram uso de Catéter de Oxigênio, 16,66\% estiveram sob Máscara de Hudson, enquanto 16,66\% necessitaram de Ventilação Mecânica Invasiva em modo PSV (Ventilação com Suporte Pressórico) (Figura 2).

Figura 2. Tipos de Suporte Ventilatório utilizados pelos pacientes pediátricos internados por COVID-19.

\section{Tipos de Suporte}

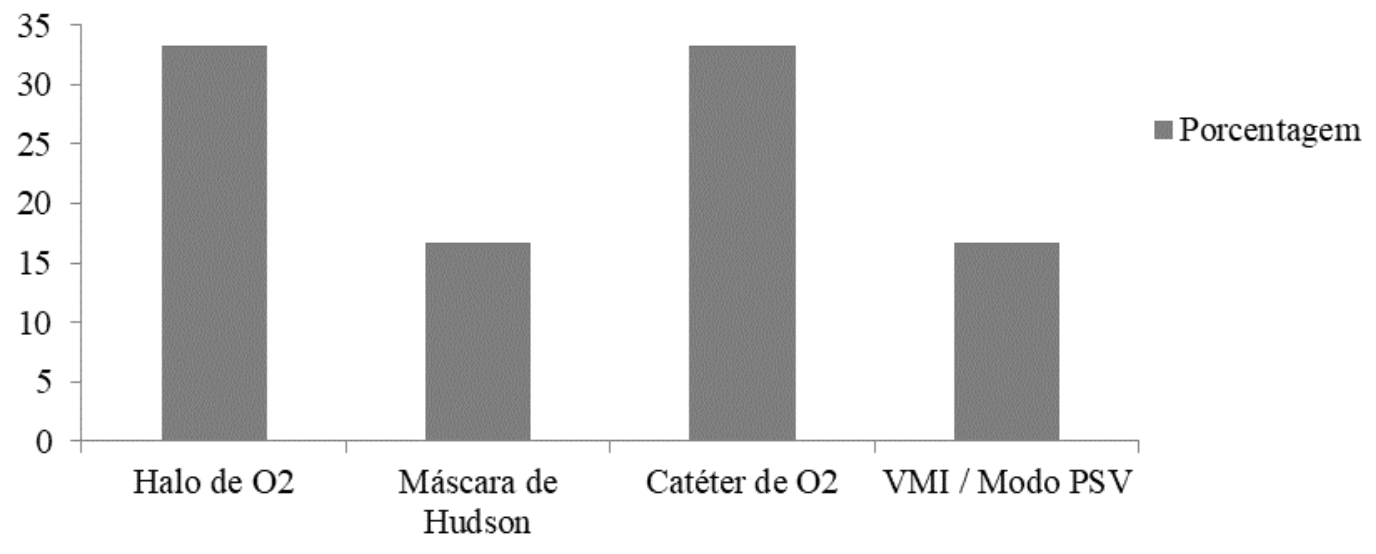

Fonte: Autores.

\section{Discussão}

Frente aos achados deste estudo, apesar de a população mais suscetível ao novo coronavírus ser principalmente adultos, idosos e pessoas com comorbidade prévia, observa-se que o vírus também está sendo causa de infecção do trato respiratório em crianças. De acordo com Safadi (2020), as razões para o risco muito menor das formas graves COVID-19 em crianças (consistentes com o que também foi observado anteriormente para surtos de coronavírus SARS e MERS), quando comparadas às faixas etárias mais velhas, permanecem incertas e várias hipóteses foram levantadas para explicar esse fenômeno, incluindo diferentes padrões de respostas imunológicas entre as idades.

O autor citado acima explica que é mais provável que os adultos, uma vez infectados com a COVID-19, desenvolvam 
respostas imunes desbalanceadas, o que leva a uma tempestade de citocinas, frequentemente associadas a danos pulmonares e pior prognóstico para os pacientes, em oposição a lactentes e crianças, nos quais seriam esperadas diferenças na imunidade inata e uma resposta mais eficiente das células $\mathrm{T}$, capazes de eliminar o vírus. Os dados disponíveis sobre a gravidade desta doença em crianças com as comorbidades são escassos, limitando a possibilidade de identificar condições com maior risco de complicações e mortalidade. Na atual pesquisa coletada no período de julho à setembro de 2020, apenas 16 crianças necessitaram de internamento na enfermaria do HUSE, constatando a baixa incidência frente a quantidade de infectados com a COVID-19 em outras faixas etárias.

Michelin (2020) afirma que uma das alterações ao exame físico é a presença de crepitações na ausculta pulmonar. O Ministério da Saúde (2020) acrescenta que a ausculta pulmonar pode revelar também estertores inspiratórios, estertores e/ou respiração brônquica em pacientes com pneumonia ou dificuldade respiratória. Observou-se na pesquisa realizada, creptos, roncos, roncos associados aos estertores crepitantes, e ausência de ruído adventício o que contribui como novos achados sobre ausculta pulmonar na SARS-CoV-2.

No presente estudo, foi identificado que $75 \%$ das crianças tiveram necessidade de fazer uso de oxigênio como medida corretora da hipoxemia. Silva; Neves; Forgiarini (2020) salientaram que ainda não há estudos randomizados ou não randomizados sobre o uso de oxigenioterapia em pacientes com COVID-19, apenas estudos em outras populações com o intuito de elaborar recomendações. Os autores demonstram em sua pesquisa que $41 \%$ de todos os doentes foram hospitalizados e mais de $70 \%$ daqueles com a forma grave da doença necessitaram de oxigênio suplementar. Para os pacientes críticos, a hipóxia pode ser prejudicial e está associada a piores resultados, levando ao aumento da morbidade. Considerando-se os prejuízos da hiperóxia e de valores elevados de $\mathrm{SpO}_{2}$, o aumento de custo em relação à maior demanda de oxigenioterapia pelos pacientes com COVID-19 e ao aumento da mortalidade nessa população com hipoxemia, recomenda-se o uso de oxigênio em pacientes com $\mathrm{SpO}_{2}<92 \%$. Como citado acima a maioria das crianças necessitaram de suplementação de oxigênio, concordando que a maioria dos pacientes apresentam essa característica.

Em relação à interface, Silva; Neves; Forgiarini (2020) ainda salientaram que não é recomendada a utilização de máscaras de Venturi para oxigenoterapia a esses pacientes. (Moderada recomendação com moderada evidência). Recomendase a utilização de cateter nasal de oxigênio com até 51/min sem ser necessária a umidificação para evitar produção de aerossóis e o risco de contaminação por outros patógenos (forte recomendação com moderada evidência) para iniciar a terapia. Pode-se utilizar a máscara cirúrgica sobre o dispositivo (fraca recomendação, com fraca evidência). Se o paciente não atingir a $\mathrm{SpO}_{2}$ alvo, recomenda-se o ajuste do fluxo de $\mathrm{O} 2$ entre 10 e 15 l/min com máscara reservatório. No vigente estudo, os tipos de suporte utilizados foram halo de oxigênio, máscara de Hudson, catéter nasal de $\mathrm{O}_{2}$ e VMI em modo PSV, sendo halo e cateter de $\mathrm{O}_{2}$ os mais aplicados.

Dias; Camelier; Dos Santos (2020) explicam que as experiências da China e da Itália demonstraram que as pessoas com COVID-19 em fase inicial da doença têm indicação mínima para o uso de técnicas de remoção de secreção brônquica, otimização da ventilação e da oxigenação. Isso foi observado com as crianças acometidas pelo novo Coronavírus, visto que apenas $37,5 \%$ apresentavam quadro secretivo.

Segundo Ferreira et al. (2020), a maioria das crianças apresentam sintomas leves, sem febre ou pneumonia. No entanto, podem manifestar sinais de pneumonia ao exame de imagem do tórax, por mais que apresentem sintomas mínimos ou inexistentes. Nesta pesquisa, 62,5\% dos pacientes evoluíram para pneumonia, associada a sintomas leves ou mais graves, corroborando os achados do estudo supracitado.

Neville; Vanzillotta; Quintão (2020) explicam que dados da China, Estados Unidos e Itália demonstraram que crianças foram responsáveis por 1 a 5\% dos casos diagnosticados por COVID-19. O grupo de pesquisa Virtual Pediatric Systems que utiliza informações de UTI pediátrica de 177 hospitais registrou, até o dia 14 de abril de 2020, 186 internações em 
UTI e três óbitos. A razão da baixa incidência de formas graves em crianças, assim como da baixíssima mortalidade nesse grupo, ainda não foi esclarecida, embora se especule que esteja relacionada à imaturidade imunológica. Tal situação justifica o desfecho das crianças internadas no Hospital de Urgência de Sergipe, uma vez que a taxa de mortalidade foi de 12,5\%.

\section{Conclusão}

Com a realização deste estudo, foi possível observar um predomínio de crianças do sexo masculino, com mediana de idade de 4 meses. Quanto às características respiratórias apresentadas pelos lactentes e crianças com testes positivos para COVID-19 submetidos ao estudo, não foram encontradas discrepâncias em relação aos sinais e sintomas apresentados pelos adultos, porém evidenciou-se que a doença acontece em menor incidência e de forma mais branda.

No entanto, faz-se necessária a realização de novos estudos relacionados ao comprometimento do vírus SARS-CoV-2 na população pediátrica, visto que, por se tratar de um vírus novo e com reações ainda pouco elucidadas, pode provocar diferentes formas de manifestação clínica, desde acometimentos mais leves a repercussões mais graves.

\section{Referências}

Governo Do Estado De Sergipe. Boletim epidemiológico para atualização sobre o COVID-19. https://todoscontraocorona.net.br/wpcontent/uploads/2020/09/Boletim-25.09.2020.pdf.

Guedes, B. L. C. d. S., Nascimento, A. K. P. d., Melo, B. T. G., Cunha, S. M. D. d., Oliveira Filho, A. A. d., \& Oliveira, H. M. B. F. d. (2020). Aspectos gerais da COVID-19 na saúde de gestantes e recém-nascidos: Uma breve revisão. Research, Society and Development, 9(7), Artigo e897974969.

Kemp, H., Corner, E. \& Colvin, L., 2020. Chronic pain after COVID-19: implications for rehabilitation. British Journal of Anaesthesia, 125(4), 436-440.

Lanza, F., Aquino, E., Sousa, M. \& Andrade, P., 2020. Protocolo de mobilização precoce de paciente crítico e reabilitação pós-alta hospitalar na população infantil acometida de COVID-19. ASSOBRAFIR Ciência, 11(Supl1), p.227.

Lingappan, K., Karmouty-Quintana, H., Davies, J., Akkanti, B. \& Harting, M., 2020. Understanding the age divide in COVID-19: why are children overwhelmingly spared?. American Journal of Physiology-Lung Cellular and Molecular Physiology, 319(1), L39-L44.

Ministério Da Saúde. Sobre a doença. https://coronavirus.saude.gov.br/sobre-a-doenca.

Neville, M., Vanzillotta, P. \& Quintão, V., 2020. Anestesiologia pediátrica e o paradoxo da COVID-19: opinião do Comitê de Anestesia em Pediatria da Sociedade Brasileira de Anestesiologia. Brazilian Journal of Anesthesiology, 70(2), pp.187-188.

Oliveira, J. P. S., Costa, A. C. B., Lopes, E. K. S., Silva, L. J. d., Gomes, K. B., Oliveira, S. d. S., \& Barros, R. P. d. (2020). Entendimento da COVID-19 sobre aspectos relacionados ao distanciamento social. Research, Society and Development, 9(10), Artigo e5739108983.

Pereira, M. D., Oliveira, L. C. d., Costa, C. F. T., Bezerra, C. M. d. O., Pereira, M. D., Santos, C. K. A. d., \& Dantas, E. H. M. (2020). A pandemia de COVID19, o isolamento social, consequências na saúde mental e estratégias de enfrentamento: uma revisão integrativa. Research, Society and Development, 9(7), Artigo e652974548.

Safadi, M., 2020. The intriguing features of COVID-19 in children and its impact on the pandemic. Jornal de Pediatria, 96(3), $265-268$.

Santos, M., Dias, C. \& Camelier, F., (2020). Atuação dos fisioterapeutas no âmbito da Atenção Primária à Saúde (APS) junto a usuários suspeitos ou diagnosticados com COVID-19: contribuições da Fisioterapia Respiratória. ASSOBRAFIR Ciência, 11(Supl1), p.31.

Silva, V., Neves, L. \& Forgiarini Junior, L., (2020). Recomendações para a utilização de oxigênio suplementar (oxigenoterapia) em pacientes com COVID-19. ASSOBRAFIR Ciência, 11(Sup11), p.87.

Smile, S. C. (2020). Supporting children with autism spectrum disorder in the face of the COVID-19 pandemic. Canadian Medical Association Journal, 192(21), E587.

Steinman, J., Lum, F., Ho, P., Kaminski, N. \& Steinman, L., (2020). Reduced development of COVID-19 in children reveals molecular checkpoints gating pathogenesis illuminating potential therapeutics. Proceedings of the National Academy of Sciences, 117(40), pp.24620-24626. 